\title{
Hydrogel-based Biosensors and Sensing Devices for Drug Delivery
}

\author{
Nicholas A. Peppas and Diana Snelling Van Blarcom \\ Institute for Biomaterials, Drug Delivery and Regenerative Medicine \\ McKetta Department of Chemical Engineering, Department of Biomedical Engineering, \\ and Division of Pharmaceutics, \\ The University of Texas at Austin, \\ Austin, Texas 78712-1062, United States
}

The Americas Special Issue of the Journal of Controlled Release

* To whom all correspondence should be addressed

Email: peppas@che.utexas.edu; Phone: (512) 471-6644 


\section{Introduction}

In the past 15 years drug delivery devices have received added attention, not only as passive systems of drug delivery that respond to the needs of the health care provider or the patient but have an added advantage or an added characteristic of being triggered by an external process of recognition of a cause, a disease or an analyte that leads to a triggering mechanism for specific drug delivery.

This is an added characteristic of new and promising systems that will, some day, respond to the surrounding environment or to early stages of a disease. The idea is not a new one but has been first presented in the classic mechanics systems of the early 1950s. We know now that in early systems there was always an input to a closed system that was somehow manipulated to lead to an output. This process was not always controlled by a simple linear function. For example, in devices or mechanical systems involving a polymeric component, a non-linear viscoelastic function would control that process. The idea was well put forward in some pioneering publications in the field. As early as 1991, Peppas and Brannon-Peppas [1] proposed that hydrogels-based sensor (of $\mathrm{pH}$ ) could be used to trigger delivery by extension of a thin $\mathrm{pH}$-sensitive polymer film. In 1996, Doyle et al [2] provided an early mathematical analysis of

one such feedback-controlled system. Though it was Heller [3] who wrote a seminal paper in which he combined all the characteristics of an effective 
mechanical and responsive system by combining feedback control, which is well known in mechanical and chemical engineering, with drug delivery.

And this led to the idea of sensors in drug delivery, systems that would naturally sense and delivery, equipped with or without mechanical parts. Such systems are characterized by two main components, a sensing device and a transducing device.

Closed loop or feedback systems can be based either on controller algorithms or on self-regulating devices. The use of a controller algorithm requires a physical implementation to interact with the physical parts of the sensor and the transducing device (actuator). Although microfabrication technology may achieve high levels of integration for a compact presentation of this type of systems, particular physical features have to be incorporated for each element producing a heterogeneous system that implies a more complex interaction with the physiological medium. A self-regulating device generally has a simpler and more homogenous configuration due to a natural and high level of integration of sensor, control and actuation functions. The main or even the only component of these systems is a smart material, that is, an environmental responsive physical element. Thus, it becomes obvious why $\mathrm{pH}$ - sensitive, temperature-sensitive or analyte-sensitive devices are of such important as sensing devices for feedback-controlled systems. And there is no better application of these systems than in treatment of diabetes by sensing glucose concentrations and delivering insulin. 
Significant developments in bionanotechnology and advanced biomaterials facilitate the design and production of such systems that not only respond to physiological conditions but act in a specific way to provide a therapeutic effect. Intelligent therapeutic delivery systems can respond to $\mathrm{pH}$, temperature, ionic strength or the concentrations of undesirable components in the body. Essentially, these processes involve a mimicking of natural responses in the body to different concentrations of hydrogel ions or target biomolecules. For example, insulin can be released in response to the sensed serum glucose level.

Numerous laboratories including ours have studied a variety of mostly non-biodegradable, biomimetic systems that establish the sensing/transducing principles. It is an effort to revolutionize medicine that we tailor therapeutics based on an individual's genetic predisposition towards disease.

Biosensors can provide feedback control by recognizing changes in its surrounding physiological or biological fluid and then taking "action", either in terms of simple movement of a device component or release of one or more drugs. In recent years we have seen an explosion in the field of such novel sensors and microfabricated devices for drug delivery [4]. Such devices provide a platform for well-controlled functions in the micro- or nano-level. They include nanoparticulate systems, recognitive molecular systems, biosensing devices, and microfabricated and microelectronic devices. The synthesis and characterization 
of biomimetic gels for drug and protein delivery systems is a significant focus of recent research.

There are numerous techniques for microfabrication of patterned polymer surfaces and microchips for medical devices [3]. While silicon has been the choice material for much of the research done with MEMs, the methacrylates and acrylates could provide an inexpensive base for future work. Several applications have already been suggested including patterned surfaces for cell adhesion, biosensors, microfluidic devices, and arrays for chemical screening.

The physicochemical understanding of such hydrogels under the conditions of application is neither simple nor well developed. Considering that all these carriers are ionic hydrogels, and that several ionic and macromolecular components are involved, with associated thermodynamically non-ideal interactions, it is evident that analysis and prediction of their swelling and response behavior is rather complex [5].

Environmentally responsive hydrogels lend themselves naturally to utilization in microfabricated devices. Polymerization of the required monomers and crosslinking agents can be done by free-radical polymerization using UV light, thus enabling photolithographic techniques to be adjusted to pattern these materials on the microscale. When hydrogels are immobilized within microstructures, the ability of the hydrogel to function is improved. There are many advantages of utilizing hydrogels in this way. First, hydrogels can be deposited permanently in specific locations of a substrate where they can exhibit 
their unique physiologically responsive characteristics repeatedly. Second, the hydrogels mechanical failure is less of an issue when the primary action is swelling against a well-defined structure.

While biosensors and sensing devices are usually made of nonbiodegradable sensing materials and substrates, environmentally sensitive hydrogel networks whose crosslinks are degradable by hydrolysis can have a significant advantage over their nondegradable counterparts. Hydrogels are capable of serving multiple functions within the body and many of these functions are only required temporarily. They are particularly useful in "feedback control devices" where an input leads to an output through a simple or complex function, unique of the polymer used. One example is a biochemical sensor, which is dependent on an enzyme.

For example, in our laboratory we have investigated the use of glucose oxidase immobilized in a swollen poly(methacrylic acid) network for the purpose of sensing glucose and responding with swelling or deswelling that can provide insulin delivery. Hydrogels can serve a dual role in sensors by first immobilizing the enzyme near the electrode surface and second, allowing the responsiveness of the gel to sense the change $[2,5]$. However, since the enzymes become inactive and degrade with time, it is desirable and more practical that the hydrogel network do the same.

Other examples of feedback control devices include responsive hydrogel nanoparticles for targeted drug delivery. We have investigated temperature- 
responsive polymer-gold nanocomposites as intelligent therapeutic systems [6]. Through spatial and temporal controlled drug delivery, injectable nanoparticle carriers have the ability to revolutionize disease treatment [7].

With the advantages of a novel degradable hydrogel network come the additional challenges of understanding the responsive swelling as the crosslinks holding together the network are being cleaved. Often, sensing elements of microsensors employ very slowly degrading hydrogel networks as sensing elements that present a useable lifetime in the device. Early studies were more concerned with mass or structural integrity losses, as many materials have been designed for structural applications in the body. However, we believe that a broader understanding of degradation is needed for these materials to be useful in new and more complex application such as biosensing. Structural integrity is much less of an issue in biosensing applications because the network is covalently attached to a rigid scaffold.

The synthesis of responsive hydrogels, which provide tunable degradation rates, is still a huge problem and a goal of the field. Put another way, it is preferred to produce hydrogels with different lifetimes as sensing elements. This is dependent among other things on the number and length of the crosslinks. Degradation occurs via a variable number of hydrolytically labile bonds in the material. The concentration of those bonds is easily changed, and thus the resulting sensing lifetime can be changed [8]. 
Let us now summarize the characteristics of complete microelectromechanical systems (MEMS) and bio- microelectromechanical systems (BioMEMS) [9]. First is the sensing element can be based on poly(methacrylic acid) or other anionic networks with hydrolytically degradable crosslinks. Of course cationically charged hydrogels can also be used as can various hybrids, composites, interpenetrating networks, etc. Poly(methacrylic acid) systems are especially interesting to biological applications because their $\mathrm{pKa}$ is so near to biological $\mathrm{pH}$. Second, the transducing element that converts the sensing element into our third required element, a measurable output, can be a piezoelectric device, a conductive polymer or even a microcantilever-based system. Microcantilevers utilize a unique sensing mechanism since they provide mechanical amplification of a signal due to change in the surface properties [10]. This heightens the sensitivity for which very small volume changes in the hydrogel can be precisely observed.

We will examine some of the pioneering work in the field below, but we will also speak on the use of biodegradable, environmentally responsive hydrogels as sensing components in novel microscale devices.

\section{$2 \quad$ Hydrogel-based biosensing}

Several decades of research have contributed to our understanding of stimuli-responsive hydrogels so that they can now be utilized in an abundance of 
sensing applications. A comprehensive knowledge of their physical and chemical properties exists along with how the materials interact with living systems. Alongside, theory has evolved to explain the unique interaction of these systems with their environment and other external stimuli. Engineers are now poised to develop and utilize novel sensing systems that allow the biological processes of life to be monitored like never before.

Hydrogels are water-swollen hydrophilic crosslinked polymers, that do not dissolve in water or biological fluids because of chemical or physical crosslinks [11]. Certain hydrogels can sense changes in their environment on a molecular level, which lead to changes in their swollen volume. These are commonly referred to as environmentally responsive hydrogels. Large changes in the swelling ratio of these hydrogels can be observed with changes in the $\mathrm{pH}$, temperature, ionic strength, nature of the swelling agent, and electromagnetic radiation [12]. Biomolecules are commonly immobilized within environmentally responsive hydrogels to yield biosensing materials. The most common of these is the immobilization of glucose oxidase within $\mathrm{pH}$-responsive hydrogels to make glucose-sensitive materials.

pH-Responsive hydrogels are anionic, cationic, or amphiphilic. Anionic hydrogels exist in a collapsed state at low $\mathrm{pH}$. When the $\mathrm{pH}$ of the external environment is raised above the $\mathrm{pK}_{\mathrm{a}}$ of the gel, they begin to swell. This is due to ionization of the side groups and repulsion of the like-charged chains. The charge repulsion is more powerful than other forces such as hydrogen bonding 
which exist between the chains in the collapsed state. At the molecular level and if the swelling is isotopic, this increase in volume corresponds to an increase in the network mesh size. Cationic hydrogels show the opposite behavior, which is swelling at low pH and collapsing at high $\mathrm{pH}$ (Figure 1).

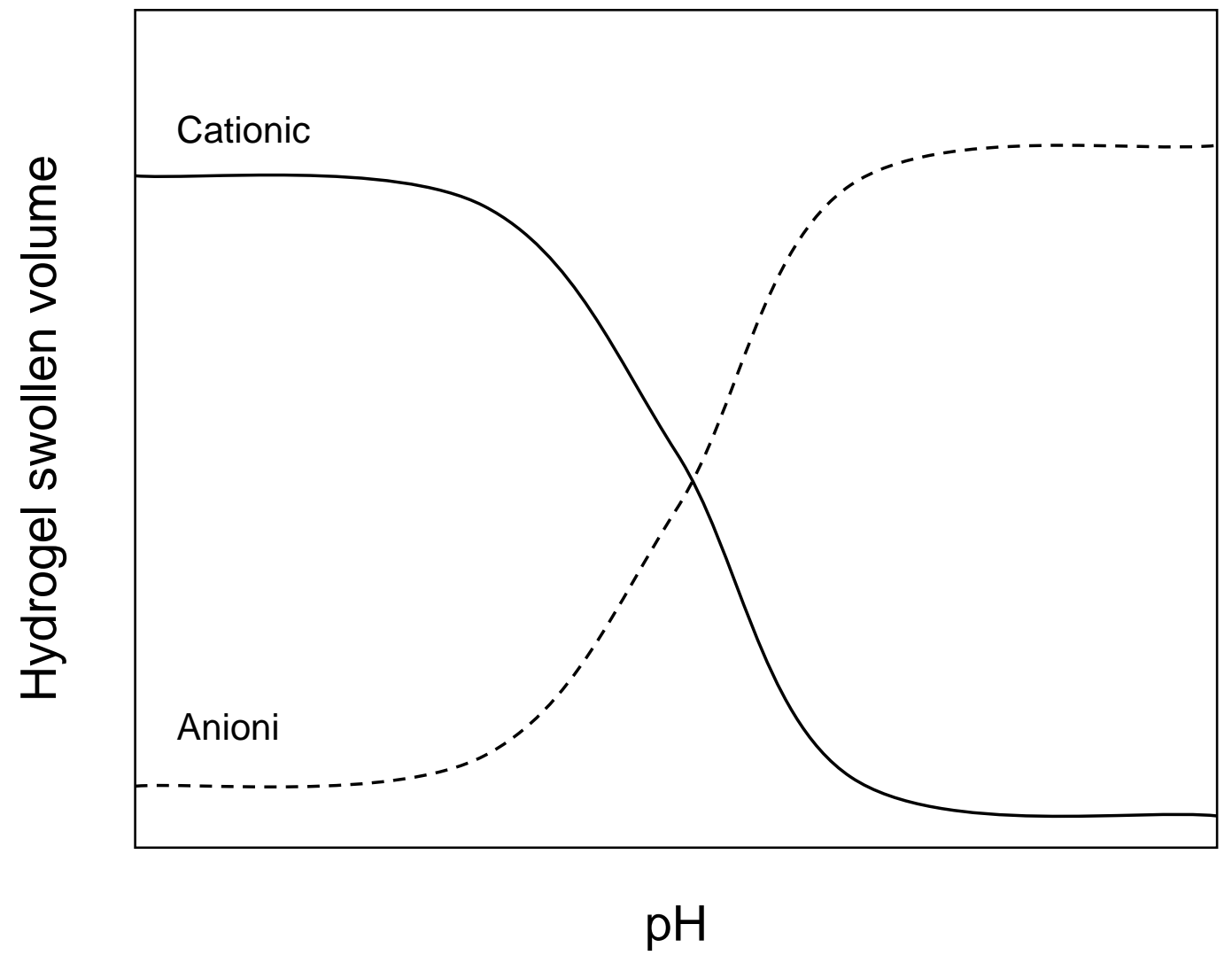

Figure 1 Cationic and Anionic pH-responsive hydrogel swelling

The reduced size of these hydrogel microstructures leads to faster mass transfer and chemomechanical response [13]. To better understand the kinetic response of hydrogel-based sensors, we must understand the kinetic response of the hydrogel itself. The swelling and shrinking of hydrogels requires transport 
of the stimulus into the network followed by water transport into or out of the network [14]. An interesting complexity in this system is that shrinking of the gel can generally occur more rapidly than its swelling. The characteristic response time in a hydrogel sensor is dependent on the square of the distance, so the hydrogel thickness should be as small as possible [15]. This is why microfabrication techniques are actively investigated with hydrogels.

It is interesting to note that the type of transducer can further slow the kinetics of the sensor. If the method of transduction is based on measurement of the changes of the optical transparency or conductivity of the material, then free swelling kinetics can apply. If the volume change of the gel must be transduced mechanically, such as by the use of microcantilevers, then the sensor will be inherently slower. The external force required slows the sensor performance versus our free swelling models [15].

\section{Application of hydrogels in biosensing}

\subsection{Hydrogels as immobilizing scaffold for biomolecules}

The first examples of hydrogels in biosensing employed hydrogels for the immobilization of biomolecules to measure biospecific interactions. In one such example, Fagerstam et al. [16] covalently attached biomolecules to thin hydrogel films atop surface plasmon resonance chips to measure biomolecular interaction kinetics and concentration. Solutions containing biomolecules of interest were flowed over the hydrogel and small mass changes were measured with a 
sensitivity of $10 \mathrm{pg} / \mathrm{mm}^{2}$. Some advantages of this early biosensor include that it did not require any biomolecular labeling and the sensor chip could be used repeatedly.

Immobilization of nucleic acids on solid supports has been widely used in the detection of DNA and other biomolecules in sensor technology. Because three dimensional hydrogel matrices offer significant advantages for capturing probes over more conventional two dimensional rigid substrates and the ability to provide a solution-mimicking environment, they are becoming increasingly attractive as desired supports for bio-analysis [17].

The use of hydrogels to immobilize enzymes for improved and sustained activity of biomolecules is not limited to the microscale. While commonly employed in lab-on-a-chip technologies, these materials also perform favorably in large-scale bioreactors as well. Hydrogel microspheres containing immobilized enzymes were used to monitor packed-bed bioreactor by Guiseppi-Elie et al. [18]. Enzyme activity was tested after the materials were stored in buffer at $4^{\circ} \mathrm{C}$ for one year. The materials retained $80 \%$ of their initial activity. This high stability shows promise towards the utilization of hydrogel-based biosensing materials on a variety of scales. Since scale-up is of common concern in the practice of chemical engineering, it is important to note that hydrogel-based biosensing elements have shown desirable performance at a variety of scales. 


\subsection{Hydrogels for improved biocompatibility of sensors}

Biofouling is one of the most significant hurdles to the advancement of novel biosensor. The innate biocompatibility and tunable permeability of hydrogels is exploited in many applications for in vivo sensing. Baxamusa et al. [19] deposited ultrathin films of poly(hydroxyethyl methacrylate) (PHEMA) by photoinitiated chemical vapor deposition (piCVD) onto bare silicon for biosensor applications. The mesh size was engineered to allow only small molecules to permeate with the exclusion of proteins that would otherwise adhere to the silicon. The coated sensor demonstrated 8-times less adhesion of bovine serum albumin (BSA) than the bare silicon surface. This piCVD method allowed the application of thin hydrogel films over sodium-sensing optodes without degradation of their response time or sensitivity.

\section{Recent Trends: The State of the Technology}

\subsection{Optical Biosensors}

Bowman et al. have used polymerization-based amplification to detect proteins and other biomolecules. Their method involves selective polymer formation only when the biomolecule of interest is present. Recently, they fabricated a novel biosensor to detect avian flu [20]. First, the sensor surface functionalized with antibody was exposed to a solution containing the analyte of interest and an antibody-functionalized photoinitiator. This resulted in the photoinitiator being bound to the substrate only when the analyte was present. 
Next, a monomer solution was introduced, and the sensor was exposed to UV light. Crosslinked hydrogel formed only when flu nucleoproteins were present. The hydrogel was visually apparent, making this simple assay one which requires no additional equipment to interpret the readout.

These sensors are particularly well suited for use in resource poor settings around the world for many reasons. They are inexpensive and robust. The photopolymerization is initiated by UV light that is easily sourced from the sun worldwide. Finally, optical biosensors whose outputs are designed to be readout by the unaided human eye, such as the one described above, is essential in third-world biosensing since additional equipment would not be available. It is important to note that the above-mentioned characteristics that benefit resource poor settings would also be beneficial in many point of care sensing devices.

Many hydrogel-based biosensors rely on a change to the equilibriumswollen volume of the hydrogel to measure chemical or biomolecular changes in the sensing environment. One such example is an optical sensor fabricated by Tierney et al. [21]. Hydrogel was adhered to the end of an optical fiber from which very sensitive swelling changes could be measured. Light was reflected at the fiber/hydrogel and hydrogel/liquid interfaces, and the difference in that length was measured and correlated to hydrogels swelling. Tierney et al. measured an acrylamide-based hydrogel at the end of the fiber with a precision of $2 \mathrm{~nm}$.

The very small hydrogel volumes utilized with this responsive hydrogel and fiber-optic transducer are critical for sensor performance. Sensor response 
times are limited by diffusion into and out of the hydrogel. With the fiber optical transducer free swelling dynamics can apply and the transducer causes no delay in sensor response. The choice of an optical fiber transducer is beneficial to the sensor's response time.

Biosensing arrays are of particular interest in the field of hydrogel-based biosensor. It is commonly proposed to produce diverse sensing arrays by photopolymerizing many different hydrogels onto the same substrate, such as silicon, forming complex biochips. Covalent adhesion of each hydrogel to a specific location on the substrate makes it possible to track the response of each hydrogel throughout testing. Meiring et al. offered an alternative to this complex method of biochip fabrication [22]. Instead of adhering the hydrogels to a common substrate, Meiring simply fabricated individual hydrogel disks with a coding system of dots. The identity of each hydrogel disk sensor was determined using pattern-recognition software. A 98\% recognition accuracy was demonstrated with nondefective sensors. This new method for generating arrays of hydrogels for biosensing would be beneficial for quick and inexpensive biochemical testing.

There is a trend in hydrogel-based biosensors to enable visual output that can be read with the unaided eye. One type of output that is particularly useful in this manner is a hydrogel that simply changes color. A dye used to optically monitor $\mathrm{pH}$ was covalently bound within the hydrogels allowing for significant color changes to be observed [23]. Fluorescent dyes are also commonly 
immobilized within a hydrogel network. A shift in the wavelength of fluorescence and the intensity of fluorescence can result from interactions between biomolecules and fluorescent molecules within the swollen hydrogel [24].

Another method to utilize fluorescence in optical biosensor involves fluorescence quenching. In one technology, uric acid was immobilized in a polyurethane hydrogel next to a metal-organic probe [25]. Fluorescence was quenched by oxygen to enable continuous optical monitoring. The biosensors produced were stable to 1 month with common interferents. These fully reversible optical biosensors demonstrated significant stability for fluorescencebased monitoring.

\subsection{Photonic Hydrogel Sensors}

Silicon-based photonic sensors have shown much promise for in vivo biosensing because of their potential for remote readout through several centimeters of tissue. However, the application of photonic sensor technologies in vivo has been limited due to the biocompatibility issues of silicon. Recently, photonic hydrogel sensors have been produced by photopolymerizing the hydrogel within the voids of a silicon photonic sensor. Wu et al. [26] fabricated a sensor to detect cholic acid from a molecularly imprinted photonic hydrogel. The MIP was photopolymerized within the voids of a colloidal crystal array. Then the silica was etched away using hydrofluoric acid to leave only photonic hydrogel. Wu observed a concentration dependent absorbance peak shift with cholic acid, which was not observed with molecular analogs dehydrocholic acid or 
deoxycholic acid. Additionally, the non-imprinted hydrogel showed no significant peak shift with all three molecules.

Similarly, Asher et al. used physically crosslinked poly(vinyl alcohol) (PVA) to make thermoreversible photonic crystals for sensor applications [27]. PVA was chosen for its biocompatibility and ability to generate thicker volumes than other photopolymerized networks. These networks were chemically modified with carboxyl or amine groups and their diffraction monitored at different $\mathrm{pH}$. A $\mathrm{pH}$-responsive hydrogel photonic sensor was demonstrated.

Maurer et al. fabricated a photonic hydrogel sensor for the detection of cholesterol [28]. A polymerized crystalline colloid array composed of poly(acrylamide-co-glycidyl methacrylate) functionalized with cholesterol oxidase was used to detect biologically significant concentrations of cholesterol in solution. A diffraction peak wavelength shift of $63 \mathrm{~nm}$ was observed, which showed promise towards use as an optical cholesterol sensor. The stability of this enzyme sensor was tested with thermal and $\mathrm{pH}$ variation, storage time, and repeated use.

\subsection{Nanoparticle-composite hydrogels for sensing}

Some hydrogel-based biosensors utilize silver or gold nanoparticles to transduce volume changes of the environmentally responsive hydrogel. As the volume swelling ratio, $\mathrm{q}$, of the hydrogel increases in response to an external stimuli, the interparticle distance of the nanoparticles increases. The interparticle distance of silver or gold nanoparticles determines the absorbance spectrum of 
the material. Noble metal nanoparticles have unique optical properties which are described by Mie theory.

In this way, Endo et al. fabricated a novel glucose sensor from silver nanoparticles and $\mathrm{pH}$-responsive hydrogel immobilized with glucose oxidase [29]. Upon exposure to glucose solution, the network swelled, producing a localized surface plasmon resonance (LSPR)-based optical biosensor. The resulting sensor showed excellent selectivity for glucose versus mannitol and sucrose.

\subsection{Gelation as a Sensing Mechanism}

Hydrogel gelation can be utilized as a sensing mechanism in certain biosensors. Knerr et al. utilized a mechanism by which hydrogelation of special peptides occurs only in the presence of zinc [30]. They proposed this hydrogel as a sensing element for sensors, which detect toxic levels of zinc pollution. The special peptide sequence which was designed for these biosensors only folds in the presence of zinc. Thus no hydrogel will form, and the protein will not fold, in an environment without zinc. This concept for the development of novel, biobased sensors can be extended to a variety of environmental conditions. Special peptides can be designed which undergo folding only when triggered by a wide variety of environmental changes, such as temperature, $\mathrm{pH}$, or ionic strength. In this way, hydrogelation based on special peptides can be utilized as a sensing mechanism in a variety of sensors. 


\subsection{Dual Monitoring from Hydrogel Sensing Element}

One method for miniaturizing the sensing elements needed to monitor bioprocessing is the creation of a dual-purpose environmentally sensitive hydrogel. Kocincova et al. synthesized a hydrogel which was responsive to both $\mathrm{pH}$ and oxygen concentration by embedding organosilica microparticles and poly(methacrylic acid)-based microbeads into polyurethane hydrogel [31]. In this way, simultaneous dual monitoring was achieved from one hydrogel. The novel dual-responsive hydrogel was used to monitor bacterial growth rates in culture with the optical readout being based on fluorescence.

\subsection{Electrical Transducers}

Recently, conductive polymers have been incorporated with environmentally responsive hydrogels to form novel biosensors. Conductive polymers possess delocalized charge along the chain backbone. Similar to their use in semiconductor manufacture, dopants are added to improve the conductivity of the polymer. Conductive polymers based on polyaniline and polypyrrole within crosslinked poly(2-hydroxyl-ethyl methacrylate) (PHEMA) hydrogels were made biospecific and demonstrated as glucose or urea sensors [32]. A simple transducer which can respond to hydrogen peroxide is beneficial coupled with glucose oxidase and other enzymes.

Research with conductive hydrogels for protein sensing has been conducted in our lab. Bayer and Peppas have utilized polyaniline (PANI) doped with a polymer acid for the purpose of transducing recognition events within a 
molecularly imprinted polymer (MIP). It is theorized that charged amino acids within the recognized protein will compete with the polymer acid and cause changes to the conductivity of the material [33]. This method for generating protein-specific sensing materials is particularly desirable because MIPs are inexpensive to synthesize and highly stable. Additionally, it should be possible to synthesize conductive MIPs responsive to a wide variety of different proteins.

Sheppard et al. microfabricated a conductimetric $\mathrm{pH}$ sensor by photopolymerizing $\mathrm{pH}$-responsive hydrogel atop planar interdigitated electrode arrays [34]. Copolymers of HEMA and N,N-dimethylaminoethyl methacrylate (DMAEMA) crosslinked with tetraethylene diacrylate were used. It was found that the conductivity of the thin hydrogel film changed as the swollen volume of the hydrogel changed in response to environmental $\mathrm{pH}$. The conductivity varied as a result of changes in the ion mobility within the hydrogel. These sensors were most sensitive around the biological $\mathrm{pH}$, about $\mathrm{pH} 7.4$, and demonstrated a linear response over the range of about $\mathrm{pH} 7$ to 8 .

\subsection{Magnetic Transducers}

Magnetoelastic sensors are of interest to biosensing primarily for their small size and remote sensing. The sensor is interrogated by a metallic field pulse, and a resulting resonance frequency is measured. Ruan coated a magnetoelastic sensor with a pH-responsive, poly(acrylic acid-co-isooctyl acrylate) hydrogel to measure small mass changes in the material [35]. $\mathrm{A} \mathrm{pH}$ resolution of $0.02 \mathrm{pH}$ was achieved over the range of $\mathrm{pH} 4.4$ to 8.5 . For sensors 
with approximately $1.4 \mu \mathrm{m}$ thickness, a response time of 120 seconds was observed.

Building up this work, Zourob et al. synthesized an organophosphorous pesticide-sensing element by immobilizing organophosphorous hydrolase within a pH-responsive hydrogel [36]. A magnetoelastic sensor was utilized as the transducer for this sensor as well. The reaction of certain pesticides with organophosphorous hydrolase caused a drop in $\mathrm{pH}$ and a change to the volume and elasticity of the hydrogel. The sensor output was monitored wirelessly via magnetic field, and the sensor was able to detect pesticides of $10^{-7}$ molar concentrations.

\subsection{Responsive hydrogel with pressure sensor chips}

Several authors have investigated the use of standard pressure sensing chips with environmentally responsive hydrogels to yield novel microsensors. Sorber et al. utilized pH-responsive poly(acrylic acid) (PAA)-based hydrogel with a piezoresistive pressure sensor chip [37]. The authors wanted to better understand the chemical changes within the hydrogel and not just the volume changes that could be transduced by the pressure chip. They used FT-IR to study chemical changes within the hydrogel and found them to be in excellent agreement with the electrical signal from the chip.

Lei et al. also utilized a pressure sensor for the transduction of $\mathrm{pH}$ responsive hydrogel, but their work was particularly interesting since the resulting sensor was capable of wireless monitoring [38]. As with other pressure-based 
hydrogel sensors, a stiff, porous membrane on one side of the material was required. A thin glass diaphragm was deflected when the hydrogel swelled causing the capacitor plates to become closer together. This resulted in a resonant frequency change which could be sensed remotely. A sensitivity of $1.16 \mathrm{MHz} / \mathrm{pH}$ was seen over the range $3.0-6.5$, and a response time of 45 minutes.

\subsection{Microcantilever-based Sensors}

Silicon-based microcantilever beams have received much attention as transducers in MEMS-based sensors. This attention is primarily due to their extreme sensitivity, very small size, and inexpensive microfabrication by methods established in the semiconductor industry. Microcantilevers can generate an optical output via the deflection of a laser beam such as in Atomic Force Microscopy (AFM) (Figure 2) or an electronic output utilizing piezoresistive microcantilevers (Figure 3). Hydrogels can be polymerized covalently to silicon microcantilevers using self-assembled monolayers (SAMs) with appropriate functionality.

Hilt et al. fabricated an ultrasensitive microsensor by micropatterning $\mathrm{pH}$ responsive poly(methacrylic acid) highly crosslinked with poly(ethylene glycol) onto silicon microcantilevers [39]. 


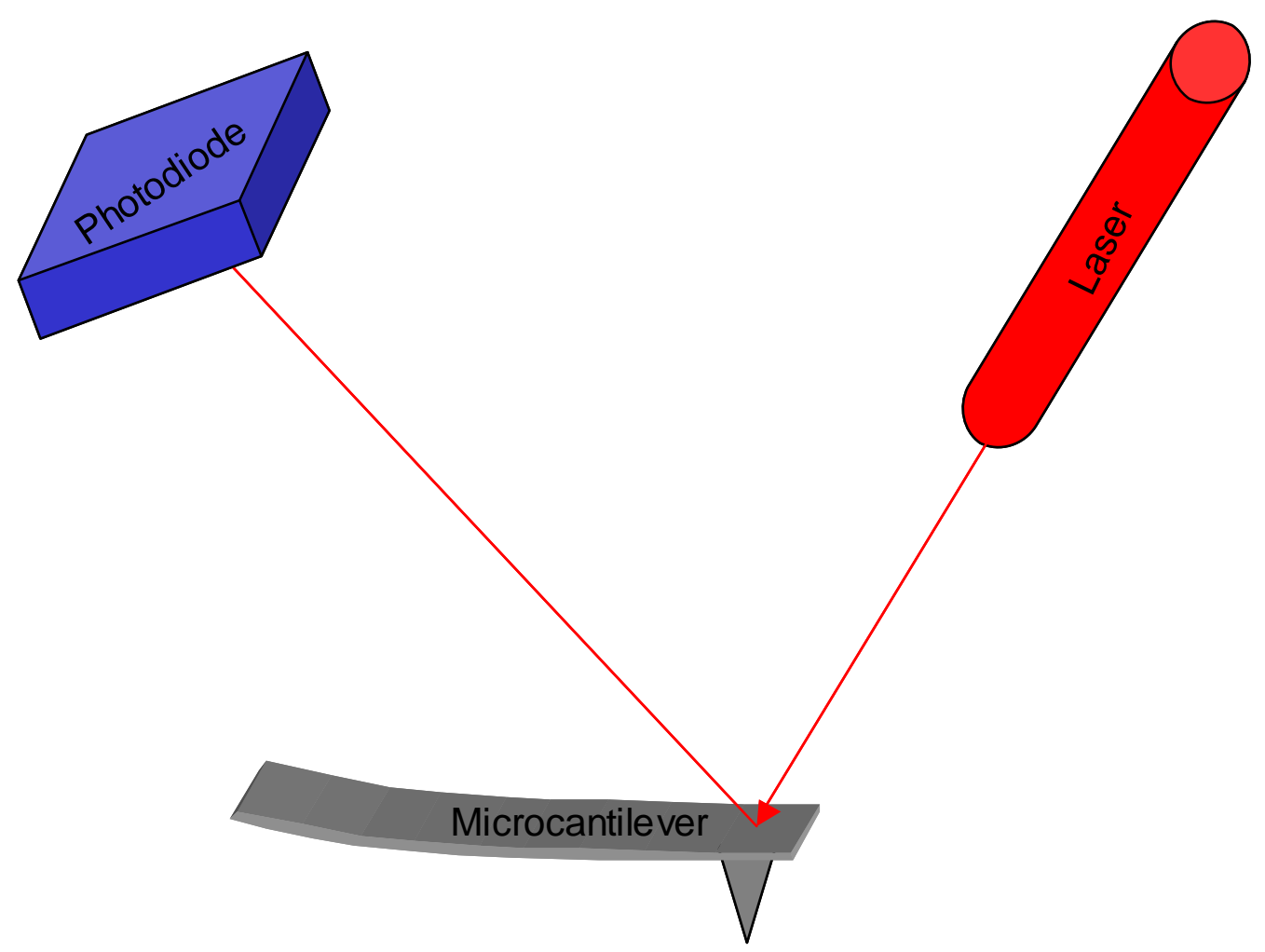

\section{Sample}

Figure 2 Microcantilever's role in Atomic Force Microscopy

A single silicon microcantilever was fabricated atop a microwell by etching (Figure 4). The hydrogel was selectively polymerized atop the beam using a photolithography mask, and the hydrogel remained adhered to the silicon surface through swelling and deswelling because an organosilane agent was used. The sensor demonstrated a maximum sensitivity of $1 \mathrm{e}-5 / \mathrm{pH}$ unit. The microcantilever-based sensors produced by Hilt et al. were most sensitive in the region just below the biological $\mathrm{pH}$ of 7.4. 


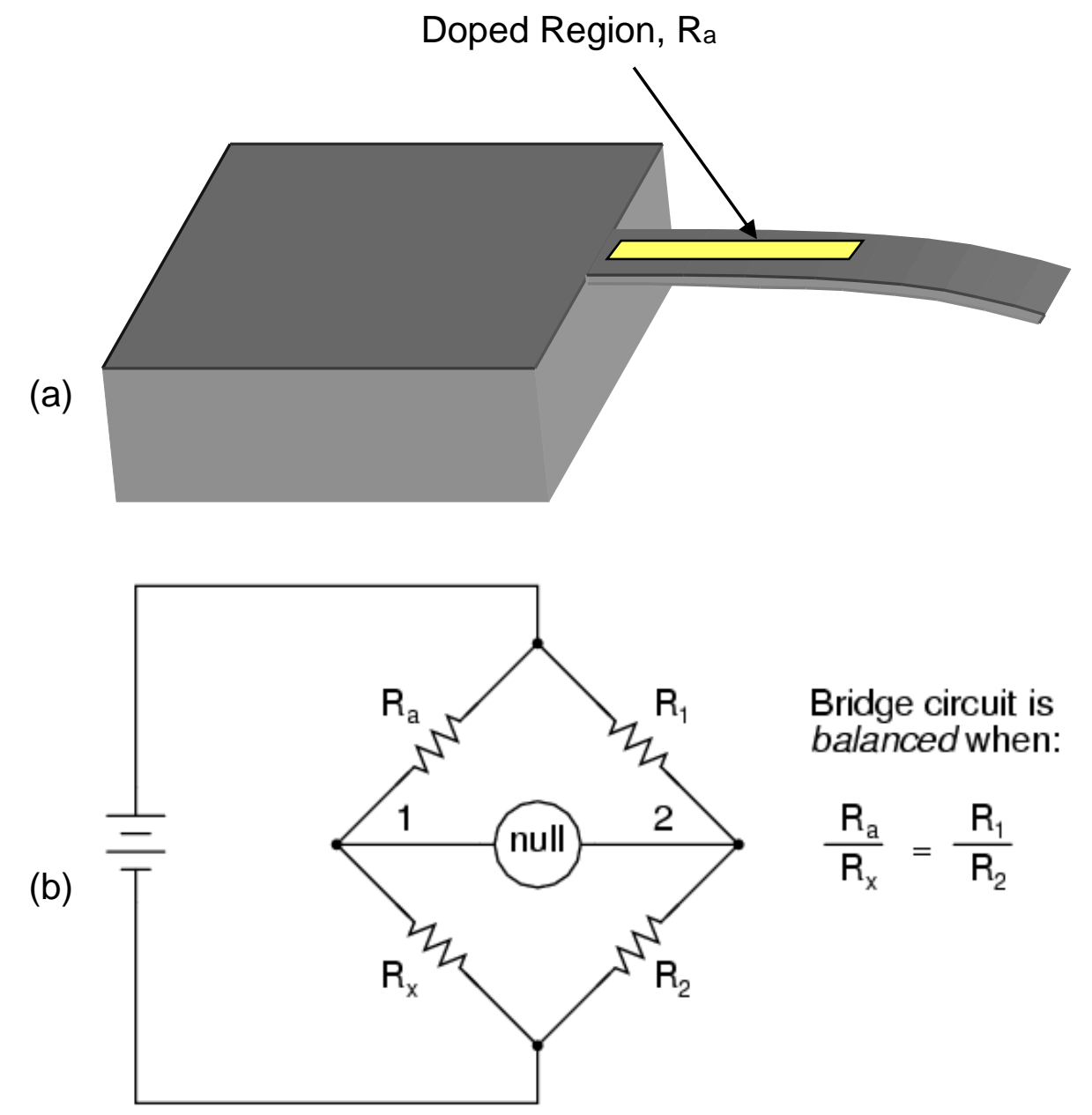

Figure 3 Piezoresistive Microcantilevers

Microcantilever-based sensors have also been fabricated by photopolymerizing a $\mathrm{pH}$-responsive hydrogel beneath the microcantilever beam. Microcantilevers of $500 \mu \mathrm{m}$ were used with a maximum deflection of $42 \mu \mathrm{m}$ observed at high $\mathrm{pH}$. Lei et al. generated a $\mathrm{pH}$ microsensor with a linear deflection in the range of $\mathrm{pH} 3$ to 6 [40]. This linear behavior was not seen in the free swelling hydrogel over the same $\mathrm{pH}$ range. An advantage of micropatterning 
the hydrogel beneath the beam is that it protects the hydrogel which lacks the mechanical strength of the silicon. A disadvantage, however, is that significantly less hydrogel surface area is exposed and thus a slower sensor response time would be expected due to diffusion limitations.

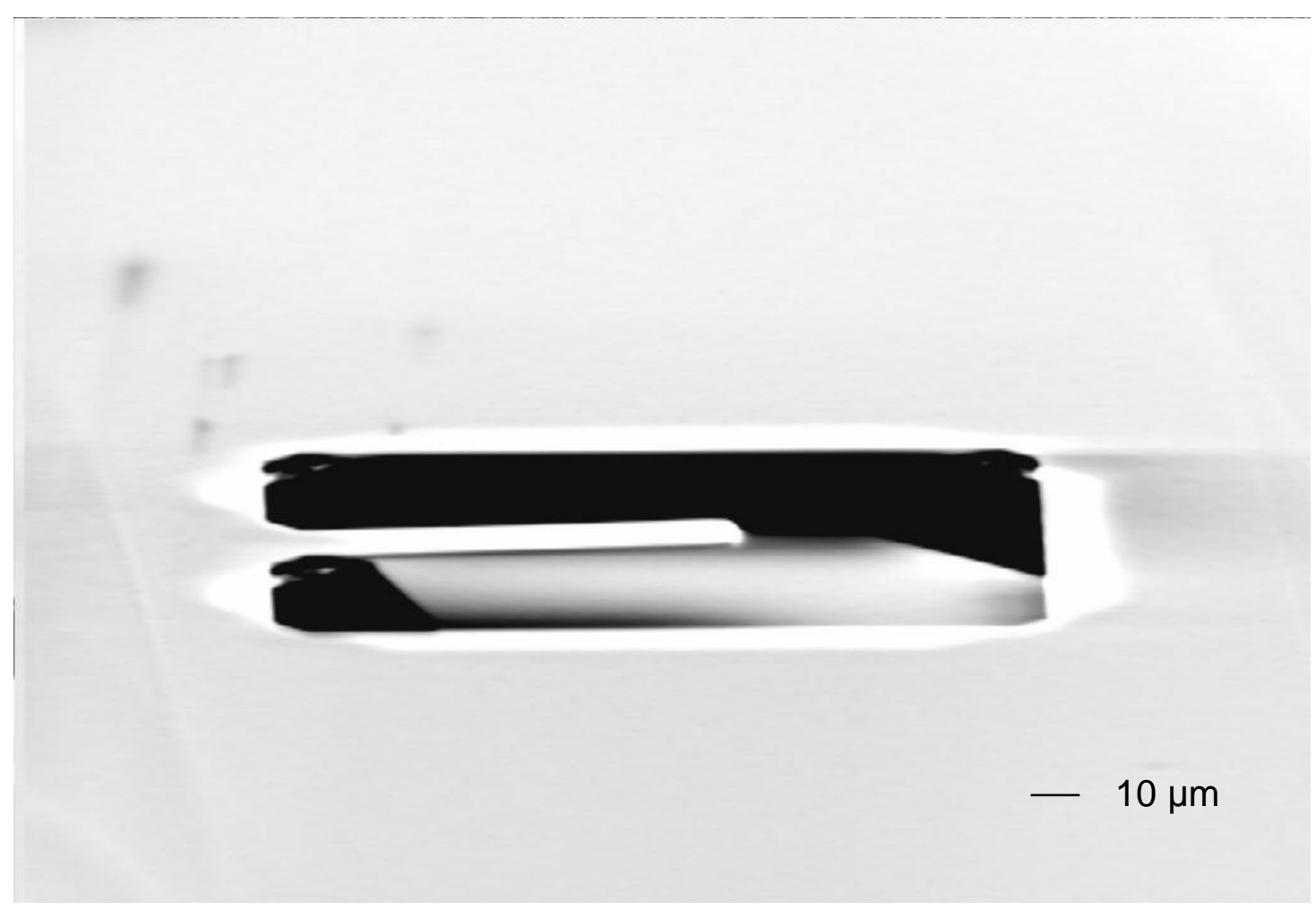

Figure 4 Single cantilever with microwell 
Since the successful transduction of environmentally-responsive hydrogels by microcantilevers has been demonstrate, it may seem that the next logical step would be to employ nanocantilevers for improved sensitivity. Nanomechanical cantilever active probes have been used for ultra small mass detection, on the order of picograms [41]. This has been achieved by measuring a shift in the piezoelectrically driven resonance frequency of the nanocantilever, and not by measuring deflection. However, the deflection of microcantilever beams by hydrogel swelling is generated by a uniform force at the interface between the silicon beam and the covalently adhered hydrogel. And thus, it should not be assumed that composite cantilevers, composed of hydrogel and silicon, would directly mimic the mass-based sensitivities observed by nanocantilevers.

\subsection{QCM-based Sensors}

Quartz crystal microbalance (QCM) have been utilized with stimuliresponsive hydrogels to generate novel microsensors for a variety of environmental conditions. A pH microsensor based on QCM was investigated by Richter [42]. A uniform film on one side of the resonator was generated by spincoating a pH-sensitive polymer mixture and then thermally initiating crosslinking to form a hydrogel. Real-time monitoring in liquid media was achieved with this microsensor. It was concluded that quartz crystal microbalances make excellent transducers for $\mathrm{pH}$-responsive hydrogels, 
primarily because they utilize thin hydrogel films, which generate the fastest responses.

A particularly interesting hydrogel-based QCM sensor was fabricated for nucleotide sensing by Kanekivo et al. [43]. Nucleotide-responsive hydrogels were utilized with QCM as a sensor for nucleotide detection. These hydrogels bound adenosine monophosphate (AMP) or adenosine triphosphate (ATP) both by boronic acid-cis-diol complexation and electrostatic interaction between the cationic unit and the phosphate group. A QCM-based sensor was fabricated from dextran hydrogels crosslinked with peptides for the detection of proteases [44]. Different protease/peptide sequence pairs were tested and the systems were found to be rather specific. Very highly crosslinked films, between $25 \%$ and 75\%, were used. This QCM-based biosensor was particularly interesting because a degradable hydrogel was utilized.

\subsection{Hydrogels in Sensing Arrays}

Hydrogels are an ideal material for use in the fabrication of diverse sensing arrays. Diverse sensing arrays are sometimes referred to as an "electric tongue" or an "electric nose", and much research has been conducted towards this end. First, hydrogels are amenable to diverse biosensing arrays since they can be easily micropatterned by photolithography and other microfabrication techniques developed by the semiconductor industry. The performance of thin hydrogel films in sensing is almost always superior to that of thicker films. The ability to use very small volumes of hydrogel is critical to the economics of 
biosensing array fabrication. While traditional monomers tend to be inexpensive, the biomolecules used in many of the responsive hydrogels presented in this chapter are not. The diversity of responsive hydrogels available for fabrication enables a bright future for hydrogels in biosensing and specifically the formation of diverse biosensing arrays.

\section{Microsensors with Si Microcantilevers and Hydrogels for Drug Delivery}

Silicon cantilevers have been used in a number of devices for detection and delivery of drugs. Several recent papers have addressed this point [45]. Typical dimensions of microcantilevers are $100 \mu \mathrm{m}$ in length by $20 \mu \mathrm{m}$ in width and $1 \mu \mathrm{m}$ in thickness. Silicon microcantilevers of these dimensions are sensitive enough to detect molecular adsorption. As a result, there has been an increased interest in their use as transducers in novel biosensors. Small size and the precision with which microcantilever deflection can be detected lend these transducers naturally to biological applications. Microcantilevers have been used to measure a wide variety of biological phenomena from the presence of specific proteins such as cholesterol in solution [46] to biological interactions such as biotin-streptavidin [47] and the hydrogen bonding of DNA base pairs [48]. Figure 5 shows one such possible application. 


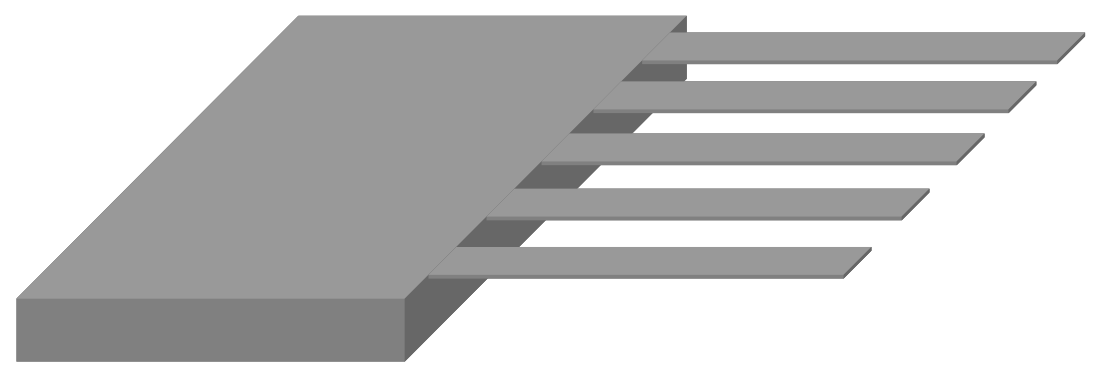

(a)

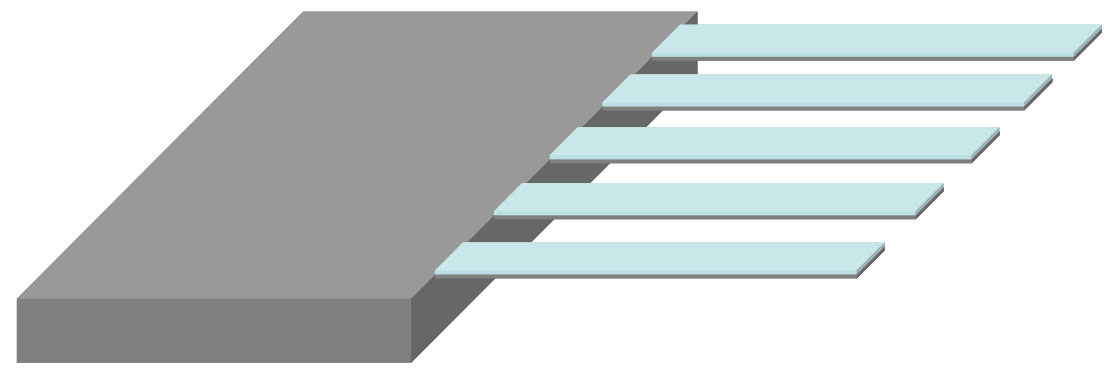

(b)

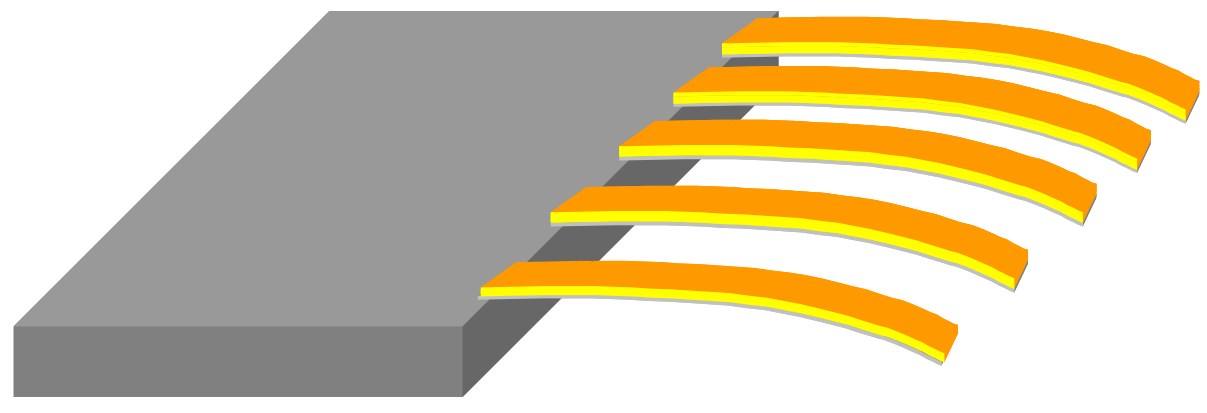

(c)

\section{Figure 5: Microcantilever arrays with responsive hydrogel}

Notably, microcantilevers are also advantageous as sensor components because they have the flexibility to work in both gaseous and liquid environments [49]. Physical, chemical, or biological stimuli can result in an electrical or optical signal when a microcantilever is used as the transducer. Cantilevers generate these signals by changes in their deflection or resonance frequency. 
An often-overlooked aspect of creating microcantilever sensors for biological applications is the application and evaluation of the thin polymer coating. Ways to characterize the coating include: comparing the resonance frequency of the bare and coated microcantilevers, SEM imaging and SFM tipscratch tests. Methods to produce micropatterned polymers on a surface include microcontact printing, laser ablation, photolithography, inkjet printing, and microscale self-assembly. One method of photolithography that does not require the use of expensive photomasks uses a commercial liquid-crystal-display projector to initiate pattern surfaces.

Hydrogel-based microcantilever sensors offer the opportunity for diverse sensing. A thin layer of a responsive hydrogel can be photopolymerized atop a microcantilever beam or a variety of responsive hydrogels photopolymerized atop the various microcantilevers in an array using a photolithographic technique. As the hydrogel responds to changes in its environment by changing its swollen volume, stress is generated at the surface between the hydrogel and the silicon. This stress results in a deflection of the beam that is both repeatable and easy to measure.

The most common method of measuring cantilever deflection is the optical method. This method uses a laser beam's deflection near the tip of the cantilever. Deflection is measured by a photodetector placed away from the cantilever. This is a preferred method of measuring deflection since it doesn't require any electrical connections and provides a linear response curve. This is 
widely considered a "simpler" method than utilizing piezoresistive readouts. Piezoresistive cantilevers have a doped region in the beam where resistivity is changed with the stress of bending. These regions are incorporated into a Wheatstone bridge so that the changes in resistivity can be measured. Other methods of producing measurable outputs include piezoelectric, capacitance and electron tunneling [45].

The sensitivity of environmentally responsive hydrogel based sensors can be ultrahigh. This is due to the fact that $\mathrm{pH}$-responsive hydrogels experience a very large change in volume over a very small change in $\mathrm{pH}$. However, the working range of these sensors is limited. They are only highly sensitive over the range where the material is partially ionized. This limited working range makes hydrogel sensors particularly well suited to biological systems since they are generally static systems where very small changes would correspond to disease states. Another advantage of these materials with regard to their sensitivity and towards their application in microscale sensors is that a 2-point calibration is all that is required. These materials generally swell linearly in their working range.

$\mathrm{pH}$-Responsive hydrogels exhibit hysteresis phenomena with respect to swelling which results in sensors that are more sensitive than they are accurate. Accuracy is difficult with these sensors because at a particular $\mathrm{pH}$ value, the swollen volume can be different whether the $\mathrm{pH}$ was arrived at by a rise in $\mathrm{pH}$ or a fall. This behavior is a result of the unique thermodynamics intrinsic to these 
materials. The narrower the $\mathrm{pH}$ fluctuations, the more accurate the value will be. This is again well suited to biological applications that would fluctuate narrowly.

The range of a hydrogel-based $\mathrm{pH}$ sensor is determined by the type of hydrogel employed in the device and its $\mathrm{pK}_{\mathrm{a}}$. The highly sensitive region of these $\mathrm{pH}$ sensors is restricted to the hydrogel phase transition range. For this reason, microscale $\mathrm{pH}$ sensors might best be employed in a microarray of similar microcantilever sensors patterned with different hydrogels. Since the $\mathrm{pK}_{\mathrm{a}}$ of a hydrogel can be tuned by the monomer choice for the gel, custom arrays are not difficult to imagine and open the field for a variety of sensing applications.

Peppas and Hilt [50] developed a microscale $\mathrm{pH}$ sensor by micropatterning poly(methacrylic acid) (PMAA) crosslinked with poly(ethylene glycol) dimethacrylate onto a silicon microcantilever. This sensor demonstrated a maximum sensitivity of $18.3 \mu \mathrm{m} / \mathrm{pH}$ units deflection. The $\mathrm{pH}$ range of the sensor was approximately 5.5-7. Another type of hydrogel-based microcantilever sensor locates the responsive hydrogel between the beam and the substrate. One advantage of this design is a greater force transfer to the beam. This sensor demonstrated a sensitivity of $7.2 \mu \mathrm{m} / \mathrm{pH}$ units over a range of 3-6 $\mathrm{pH}$ units. Another advantage is that the $\mathrm{pH}$ range would not be restricted by the beam colliding with the silicon substrate. However, one notable disadvantage of this design is that polymerization with photolithography would be limited when the hydrogel is polymerized beneath a silicon beam. 
With cantilever sensors, smaller is not always better, as the size of the sensor must be chosen based on the output desired. Nanocantilevers can translate extremely small changes in mass into large changes in their frequency. While nanocantilevers have the advantage of measuring very small mass changes, these are not usually necessary in hydrogel-based sensors because hydrogels experience a very large volume and thus mass change over a small $\mathrm{pH}$ range. Another way to engineer these systems is by utilizing analogous microstructures such as bridges and cantilever-like structures with more than one anchor point, rather than the standard cantilever beam.

As an example, sensing arrays composed of 8 microcantilevers of type 5 (Table 6.1) photopolymerized with poly(methacrylic acid) crosslinked with polycaprolactone diacrylate [45] were tested in a range of phosphate-citrate buffer solutions of constant ionic strength (0.5M). In one study, the average deflection of all cantilevers in the array was measured in order of high to low $\mathrm{pH}$ with 2 hour equilibration times (Figures 6,7 and 8). The difference in tip deflection between $\mathrm{pH} 4$ and $\mathrm{pH} 7.5$ was approximately 40 microns.

The sensitivity of novel $\mathrm{pH}$ microsensors has been tested in $10 \% \mathrm{FBS} \mathrm{pH}$ buffer solutions of constant ionic strength. The average deflection of microcantilever-based sensors prepared by Snelling and Peppas [45] in the array versus $\mathrm{pH}$ is shown in Figure 8. A hyperbolic tangent fit of the data produced the dotted line in the figure. From this data, we observe a reduction in the maximum deflection of the cantilevers observed at high $\mathrm{pH}$. An approximately $25 \%$ 
reduction in the sensitivity of these sensors was observed, but overall the presence of proteins in solution did not compromise the function of the sensing element.

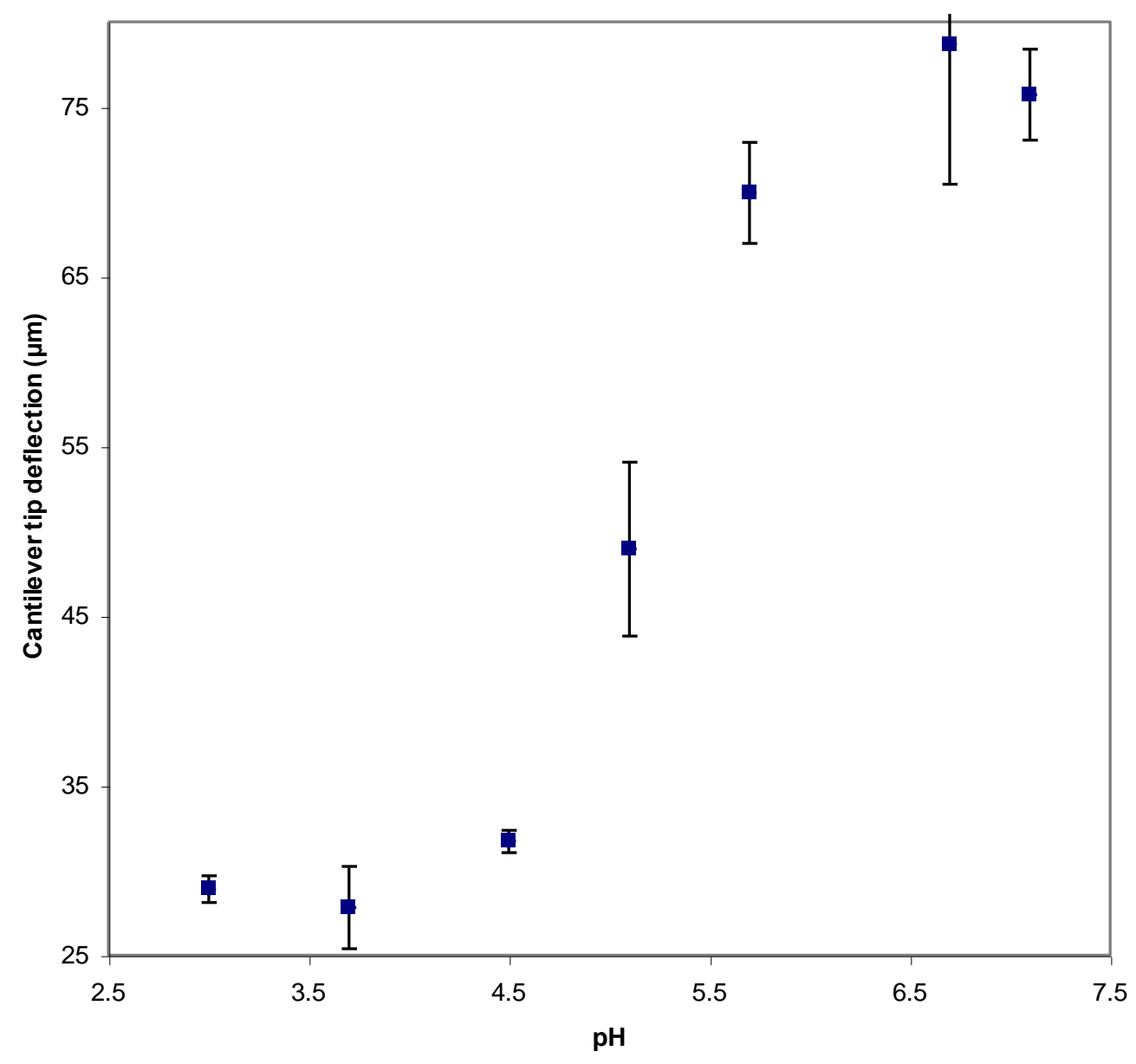

Figure 6: Microcantilever with $\mathrm{pH}-$-responsive hydrogel atop beam 


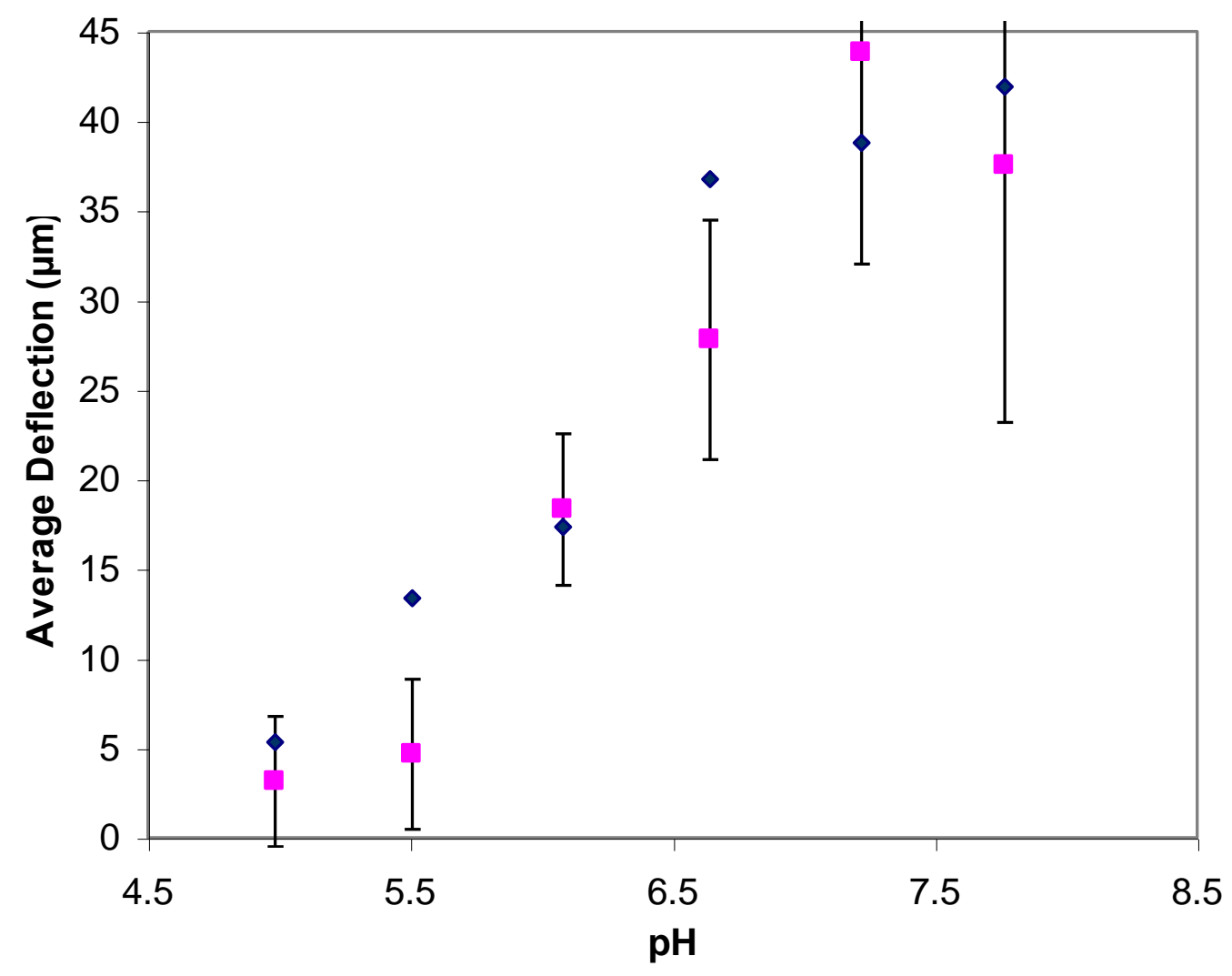

Figure 7: Average deflection versus $\mathrm{pH}$ with different equilibration times 


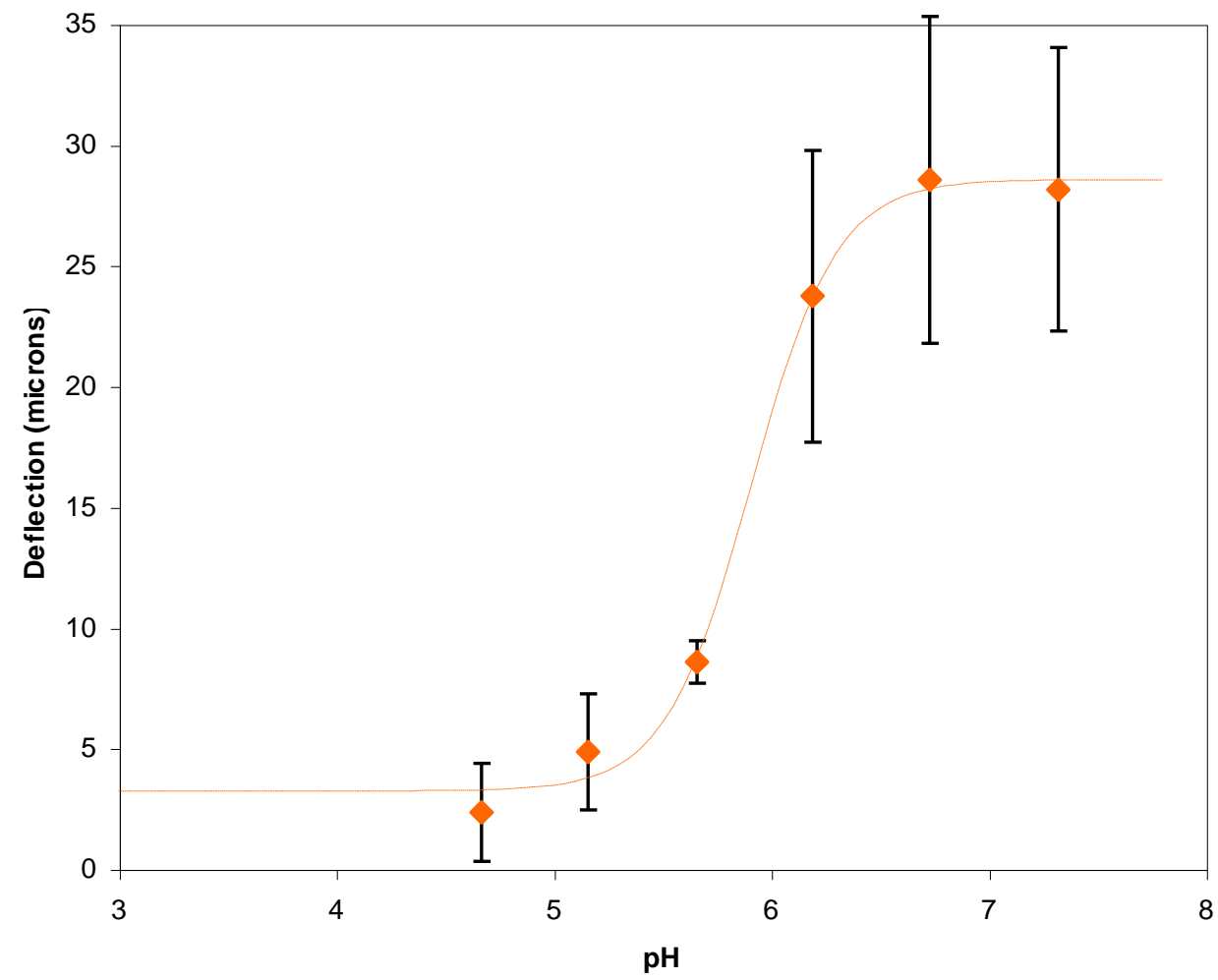

Figure 8: Microcantilever deflection versus $\mathrm{pH}$ in serum

\section{Conclusions and the future for Hydrogels in Biosensing}

It is evident that hydrogels have a bright future in the field of biosensing and specifically for the fabrication of microscale biosensors. Hydrogels have grown beyond their early uses as a scaffold for the immobilization of biomolecules and to discourage protein adhesion and promote biocompatibility of sensors. Now many stimuli responsive hydrogels have been utilized as a direct means of sensing a variety of environmental conditions. A wide variety of 
techniques have been demonstrated to transform the volume change experienced by stimuli-responsive hydrogels into an easy-to-read signal. With the aid of these transducers, hydrogel-based microsensors can generate outputs such as optical, electronic, and magnetic. We believe that the most promising application of hydrogels in biosensing will be their use in diverse sensing arrays.

\section{Acknowledgments}

This work was supported by a grant form the National Science Foundation and an NSF Fellowship to DSVB. We thank Dr. Amey Puranik for important technical assistance. 


\section{References}

1. L. Brannon-Peppas and N.A. Peppas, Time-Dependent Response of Ionic Polymer Networks to $\mathrm{pH}$ and lonic Strength Changes, Intern. J. Pharmac., 70, 53-57 (1991).

2. Doyle, F.J. III, Dorski, C., Harting, J.E., Peppas, N.A., Control and modeling of drug delivery devices for the treatment of diabetes. Proceed. Amer. Control Confer., 1996: 776-780.

3 Heller, A., Integrated Medical Feedback Systems for Drug Delivery, AIChE Journal, 51, 1054-1066 (2005)

4. Bayer, C.L. and Peppas, N.A., Advances in recognitive, conductive and responsive delivery systems. J Controlled Release, 2008, 132, p 216-221.

5. Kryscio, D. R., and Peppas, N.A., Critical Review and Perpective of Macromolecularly Imprinted Polymers , Acta Biomaterialia, 2012, 8, p 461477.

6. Owens, D.E., Eby, J.K., Jian, Y., and Peppas, N.A., Temperatureresponsive polymer-gold nanocomposites as intelligent therapeutic systems. J Biomed Mater Res A, 2007. 83A: p. 692-695.

7. Li L., Shi, Y., Pan, L., Shi Y., and Yu G, , Rational Design and Applications of Conducting Polymer Hydrogels as Electrochemical Biosensors. J. Mater. Chem.B. , 2015. 3: p. 5111-5121.

8. Chao, G.T., Qian Z.Y., Huang, M.J., Kan, B., Gu Y. Chun, Gong C. Y., Yang, J. L., Wang K., Dai, M., Li X. Y., Gou M.L. Tu M.J. and Wei Y. Q.., 38 
Synthesis, characterization, and hydrolytic degradation behavior of a novel biodegradable $\mathrm{pH}$-sensitive hydrogel based on polycaprolactone, methacrylic acid, and poly(ethylene glycol). J Biomed Mater Res A, 2008. 85A(1): p. 36-46.

9. Buerk, D.G., Biosensors, Theory and Applications. 1993, Lancaster, PA: Technomic Publishing Company, Inc.

10. Hilt JZ, Gupta AK, Bashir R, Peppas NA. Ultrasensitive BioMEMS sensors based on microcantilevers patterned with environmentally responsive hydrogels. Biomed Microdev. 2003, 5, 177-184.

11. Lowman, A.M. and N.A. Peppas, Solute transport analysis in $\mathrm{pH}-$ responsive, complexing hydrogels of poly(methacrylic acid-g-ethylene glycol). J Biomat Sci-Polym E, 1999. 10(9): p. 999-1009.

12. Peppas, N.A., Hilt J.Z., Khademhosseini A., Langer R., Hydrogels in biology and medicine: From molecular principles to bionanotechnology. Adv Mater, 2006. 18(11): p. 1345-1360.

13. Lei, M., Ziaie B., Nuxoll, E, Kristof I, Noszticius Z, Siegel R.A., Integration of hydrogels with hard and soft microstructures. J Nanosc Nanotechn, 2007. 7(3): p. 780-789.

14. M.P.M. Dicker, Bond, I.P., Rossiter J.M., Faul C.F.and Weaver P.M. . Modelling and Analysis of $\mathrm{pH}$-Responsive Hydrogels MRS Proceedings, 2015. 1718: published on line, DOI: http://dx.doi.org/10.1557/opi.2015.20 
15. Richter, A., PAschew G., Klatt S., Liening J, Arndt K.F., Adler H.J.., Review on hydrogel-based pH sensors and microsensors. Sensors, 2008. 8: p. 561-581.

16. Fagerstam, L.G., Frostell-Karlsson, A., Karlsson, R., Persson, B., Ronnberg, I., Biospecific interaction analysis using surface-plasmon resonance detection applied to kinetic binding-site and concentration analysis. J Chromatogr, 1992. 597: p. 397-410.

17. Tang, J., Xiao, P.F., Polymerizing immobilization of acrylamide-modified nucleic acids and its applications. Biosens Bioelectron, 2009. 24(7): p. 1817-1824.

18. Guiseppi-Elie, A., Sheppard Jr., N.F., Brahim, S., Narinesingh, D., Enzyme microgels in packed-bed bioreactors with downstream amperometric detection using microfabricated interdigitated microsensor electrode arrays. Biotechno Bioeng, 2001. 75(4): p. 475-484.

19. Baxamusa, S.H., Montero, L., Dubach, J.M., Clark, H.A., Borros, S., Gleason, K.K., Protection of sensors for biological applications by photoinitiated chemical vapor deposition of hydrogel thin films. Biomacromolecules, 2008. 9(10): p. 2857-2862.

20. Sikes, H.D., Jenison R., Bowman C.N., Antigen detection using polymerization-based amplification. Lab Chip, 2009. 9(5): p. 653-656. 
21. Tierney, S., Hjelme D.R., Stokke, B.T., Determination of swelling of responsive gels with nanometer resolution. Fiber-optic based platform for hydrogels as signal transducers. Anal Chem, 2008. 80(13): p. 5086-5093.

22. Meiring, J.E., Lee, S., Costner, E.A., Schmid, M.J., Michaelson, T.B, Wilson, C.G., Grayson, S.M., Pattern recognition of shape-encoded hydrogel biosensor arrays. Opt Eng, 2009. 48(3).

23. Mohr, G.J., Muller, H., Bussemer, B., Stark, A., Carofiglio, T., Trupp, S., Heuermann, R., Henkel, T., Escudero, D., Gonzalez, L., Design of acidochromic dyes for facile preparation of $\mathrm{pH}$ sensor layers. Anal Bioanal Chem, 2008. 392(7): p. 1411-1418.

24. Thete, A.R., Henkel, T., Gockeritz, R., Endlich, M., Kohler, J.M., Grob, G.A., A hydrogel based fluorescent micro array used for the characterization of liquid analytes. Anal Chim Acta, 2009. 633(1): p. 81-89.

25. Schrenkhammer, P., Wolfbeis, O.S., Fully reversible optical biosensors for uric acid using oxygen transduction. Biosens Bioelectron, 2008. 24(4): p. 994-999.

26. Wu, Z., Direct and label-free detection of cholic acid based on molecularly imprinted photonic hydrogels, J Mater Chem, 2008. 18: p. 5452-5458.

27. Asher, S.A., Kimble, K.W., Walker, J.P., Enabling thermoreversible physically cross-linked polymerized colloidal array photonic crystals. Chem Mater, 2008. 20(24): p. 7501-7509. 
28. Maurer, M.K., Gould, S.E., Scott, P.J., Cholesterol oxidase functionalization of a polymerized crystalline colloidal array. Sensor Actuat B-Chem, 2008. 134(2): p. 736-742.

29. Endo, T., Ikeda, R., Yanagida, Y., Hatsuzawa, T., Stimuli-responsive hydrogel-silver nanoparticles composite for development of localized surface plasmon resonance-based optical biosensor. Anal Chim Acta, 2008. 611(2): p. 205-211.

30. Knerr, P.J., Micklitsch, C.M., Thorpe, C., Schneider, J.P., Zinc-triggered hydrogelation of designed beta-hairpin peptides. Biopolymers, 2007. 88(4): p. 639.

31. Kocincova, A.S., Nagal, S., Arain, S., Krause, C., Borisov, S.M., Arnold, M., Wolfbeis, O.S., Multiplex bacterial growth monitoring in 24-well microplates using a dual optical sensor for dissolved oxygen and $\mathrm{pH}$. Biotechnol Bioeng, 2008. 100(3): p. 430-438.

32. Brahim, S., Wilson, A.M., Narinesingh, D., Iwuoha, E., Guiseppi-Elie, A., Chemical and biological sensors based on electrochemical detection using conducting electroactive polymers. Microchim Acta, 2003. 143(2): p. 123137.

33. Bayer, C.L. and N.A. Peppas, Advances in recognitive, conductive and responsive delivery systems. J Control Release, 2008. 
34. Sheppard, N.F., Lesho, M.J., McNally, P., Francomacaro, A.S., Microfabricated conductimetric pH sensor. Sensor Actuat B-Chem, 1995. 28(2): p. 95-102.

35. Ruan, C.M., Zeng, K., Grimes, C.A., A mass-sensitive pH sensor based on a stimuli-responsive polymer. Anal Chim Acta, 2003. 497(1): p. 123131.

36. Zourob, M., Ong, K.G., Zeng, K., Mouffouk, F., Grimes, C.A., A wireless magnetoelastic biosensor for the direct detection of organophosphorus pesticides. Analyst, 2007. 132(4): p. 338-343.

37. Sorber, J., Steiner, G., Schulz, V., Guenther, M., Gerlach, G., Salzer, R., Arndt, K., Hydrogel-based piezoresistive $\mathrm{pH}$ sensors: Investigations using FT-IR attenuated total reflection spectroscopic Imaging. Anal Chem, 2008. 80(8): p. 2957-2962.

38. Lei, M., Ziaie, B., Nuxoll, E., Ivan, K., Noszticzius, Z., Siegel, R.A., Integration of hydrogels with hard and soft microstructures. J Nanosci Nanotechno, 2007. 7(3): p. 780-789.

39. Hilt, J.Z., Gupta, A.K., Bashir, R., Peppas, N.A., Ultrasensitive biomems sensors based on microcantilevers patterned with environmentally responsive hydrogels. Biomed Microdevices, 2003. 5(3): p. 177-184.

40. Salehi-Khojin A., Bashash, S., Jalili, N., Muller, M., Berger, R., Nanomechanical cantilever active probes for ultrasmall mass detection. $\mathrm{J}$ Appl Phys, 2009. 105(1). 
42. Richter, A., Bund, A., Keller, M., Arndt, K., Characterization of a microgravimetric sensor based on $\mathrm{pH}$ sensitive hydrogels, Sensor Actuat B-Chem, 2004. 99(2): p. 579-585.

43. Kanekiyo, Y., Sano, M., Iguchi, R., Shinkai, S., Novel nucleotideresponsive hydrogels designed from copolymers of boronic acid and cationic units and their applications as a QCM resonator system to nucleotide sensing. J Polym Sci A1, 2000. 38(8): p. 1302-1310.

44. Stair, J.L., Watkinson, M., Krause, S., Sensor materials for the detection of proteases. Biosens Bioelectron, 2009. 24(7): p. 2113-2118.

45. D. Snelling Van Blarcom and N.A. Peppas, Microcantilever Sensing Arrays from Biodegradable, pH-Responsive Hydrogels, Biomedical Microdevices, 13, 829-836 (2011).

46. Boisen, A. and Thundat, T., Design \& fabrication of cantilever array biosensors. Mater Today, 2009. 12(9): p. 32-38.

47. Moulin, A.M., O'Shea, S.J., Welland, M.E., Microcantilever-based biosensors. Ultramicroscopy, 2000. 82(1-4): p. 23-31

48. Chilkoti, A., Boland, T., Ratner, B.D., Stayton, P.S., The relationship between ligand-binding thermodynamics and protein-ligand interaction forces measured by atomic force microscopy. Biophys J, 1995. 69(5): p. 2125-30. 
49. Boland, T., Ratner, B.D., Direct measurement of hydrogen bonding in DNA nucleotide bases by atomic force microscopy. Proc Natl Acad Sci USA, 1995. 12: p. 5297-301.

50. Hilt, J.Z., Gupta, A.K., Bashir, R., Peppas, N.A., Ultrasensitive biomems sensors based on microcantilevers patterned with environmentally responsive hydrogels. Biomed Microdevices, 2003. 5(3): p. 177-184. 


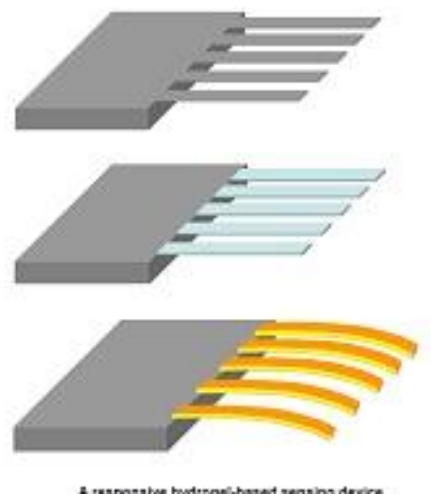

